\title{
Male sterility in potato - perspectives for developing hybrid seed breeding
}

Gavrilenko T. ${ }^{1,2 *}$, Anisimova I. ${ }^{1}$, Shishova M. ${ }^{2}$, Antonova O. ${ }^{1}$

${ }^{1}$ N.I. Vavilov All-Russian Institute of Plant Genetic Resources (VIR), St. Petersburg, Russia

${ }^{2}$ St. Petersburg State University, St. Petersburg, Russia

*e-mail: tatjana9972@yandex.ru

Common potato (Solanum tuberosum L.) is the most important non-grain crop. Until recently vegetative propagation by tubers has been the main method of potato cultivation. A shift of interest to sexual potato reproduction by true botanical seeds is due to the appearance of a new hybrid seed breeding strategy. The main expected advantages of this strategy is a decrease in the risk of plant contamination because most potato pathogens are not transmitted with pollen and seeds. Successful application of $\mathrm{F}_{1}$ hybrid seed breeding for many other crops was supported by cytoplasmic male sterility and/or by nuclear(genic) male sterility which allow to avoid emasculation.

Eight cytoplasmic types can be distinguished in potato with DNA markers: A, M, P, W/beta, W/alpha, W/gamma, D, T; three last types are known as sterile which exhibit a different phenotypic appearance of male sterility. This investigation is focused on the study of cytoplasmic types in Russian potato gene pool and estimation the perspectives of their application for developing hybrid seed breeding.

Three major sterile cytoplasm types have been detected in the subset of $\sim 220$ Russian potato varieties: T ( 43\%), D ( 47\%), W/gamma ( 10\%).

According to the results of pollen stainability test, many of varieties with $\mathrm{T}$ and $\mathrm{D}$ sterile cytoplasm types had a high pollen fertility level. Some of them were used as effective pollinators that indicating possible presence of functional alleles of nuclear fertility Restorer genes. Analysis of polymorphism in the sequences of RFL-PPR gene homologs in genotypes with $\mathrm{T}$ and $\mathrm{D}$ cytoplasm types demonstrate that the analyzed sequences belong to the $R F L-P P R$ gene subfamily and may be considered as $R f$ gene candidates in potato.

In contrast, genotypes with W/gamma_sto cytoplasm had always sterile pollen and were characterized by male tetrad sterility when tetrads fail to disintegrate in microspores. The data of metabolite profiling of the anthers in fertile and male sterile (W/gamma_sto) genotypes support the nuclear-cytoplasmic male sterility postulated earlier for potato. 